astrocytoma of the cerebral hemispheres. $\mathcal{f}$ Neurosurg 1984;61:665-73.

2 Piepmeier JM. Observations on the current treatment of low-grade astrocytic tumors of 1987;67:177-81.

3 Smith DF, Hutton JL, Sandermann D, Foy PM, Shaw MDM, Williams IR, Chadwick DW. The prognosis of primary intracerebral tumours presenting with epilepsy: the outcome of medical and surgical management. $\mathcal{F}$ Neurol Neurosurg Psychiatry 1991;54:915-20.

4 Wilden JN, Kelly PJ. CT computerised stereotactic biopsy for low density CT lesions presenting with epilepsy. $\mathcal{F}$ Neurol Neurosurg Psychiatry 1987;50:1302-5.

$D$ Smith et al reply:

We thank Mr Wilden for his interest in this article. Whilst recognising minor problems with some of our definitions, we would like to emphasise that this was a retrospective study which generates a simple hypothesis demanding prospective testing.

We agree that definitions of "early" and "late" surgery have not been agreed upon and that our own definitions, of necessity, are somewhat arbitrary. They do, however, allow the essential differentiation between those patients receiving surgery soon after diagnosis and those in whom surgery is delayed until a change in clinical status occurs. Furthermore, by using an intention to treat analysis, we avoided bias against the effectiveness of surgery as a whole by excluding patients whose surgery was performed because of neurological deterioration.

With regard to the type of surgeryresective surgery refers to procedures which involve debulking of the tumour and nonresective to biopsy or drainage of cysts. As $\mathrm{Mr}$ Wilden states, we have not subdivided resective surgery according to whether or not the surgeon considered the resection to be complete or partial. However, this information is difficult to obtain from a retrospective review of case notes and is not likely to be reliable. Furthermore, whilst accepting that this issue may be relevant in a minority of patients, with well circumscribed temporal or frontal lobe tumours, it is well recognised that resections "considered" to be total macroscopically are rarely confirmed on histopathological specimens, and many pragmatic neurosurgeons would concede that total excision of infiltrative gliomas, often involving more than one lobe, is not possible.

Mr Wilden has answered his second query himself. We would, however, like to emphasise that it was never our intention to conduct sub-group analyses, in small numbers of patients, and it is well known that such analyses on retrospective data yield unreliable results that are very difficult to interpret.

His final remarks merit two comments. Firstly, we would like to reiterate that we made every effort not to over-interpret our data and our intention was simply to generate a testable hypotheses and certainly not the development of "definitive management policies". Secondly, we must take issue with $\mathrm{Mr}$ Wilden's comments on the need for national database derived from individual centres inevitably employing different treatment regimens. Data collected in this way would not allow development of satisfactory management policies and the necessary information can only be obtained, as we state clearly in our final paragraph, from a well designed, multi-centre, prospective randomised trial comparing conservative and aggressive management policies.

\section{Extracranial vertebral artery dissection}

We would like to make several comments about the recent report by Hinse et $a^{1}$ on extracranial vertebral artery (VA) dissection: 1) This article is based on 4 personal cases and a review of 53 published cases. However, several published reports, including 2 recent studies $^{23}$ on a total of 38 cases, are not quoted, which may give the impression that mated that nearly a hundred cases of VA dissections had appeared in the English language literature in the past decade and that between 0.5 and 2.5 cases per year were reported from large referral-based hospitals. own work on the subject ${ }^{2}$ regarding symptoms and prognosis of this disorder. The authors, however, do not mention in their discussion that VA dissection may be asymptomatic and possibly discovered when exploring a concomitant carotid artery dissection. They point out that there is a high incidence of bilateral VA dissection but do not mention the possibility of simultaneous occurrence of vertebral and internal carotid artery dissections, which is not infrequent particularly in patients with spontaneous dissection. ${ }^{23}$ The frequency of multivessel dissection implies that four-vessel exploration (by angiography VA dissection is rare. In 1988, $\mathrm{Hart}^{4}$ esti-

2) We have come to similar conclusions in our

or other method) should always be attempted if a VA dissection is demonstrated.

3) The diagnosis of dissection relies classically on angiography, which reveals irregular stenosis, pseudoaneurysm, occlusion, or double-lumen. Of these features, the only pathognomonic one (but also the rarest) is the double lumen. Occlusion which can be due to thrombosis of any cause is the least specific. We think that the diagnosis of probable occlusive form of VA dissection can only be made when there is angiographic evidence of dissection in the other VA or in the internal carotid artery. In this respect, the diagnosis of dissection in patients 2 and 3 of Hise et al ${ }^{1}$ who had VA occlusion should only be regarded as possible.

4) Normalisation or improvement of angiographic abnormalities is frequent in this condition and is an excellent argument in favour of the diagnosis. In one of our cases, ${ }^{2}$ marked improvement was observed as early as 7 days after the first angiogram, which stresses the point that the first angiography should be performed as early as possible so that the diagnosis should not be overlooked. We also concluded that control angiography could be performed around the third month, a time by which most dissected vertebral arteries had returned to normal.

5) In our opinion, ultrasound examination
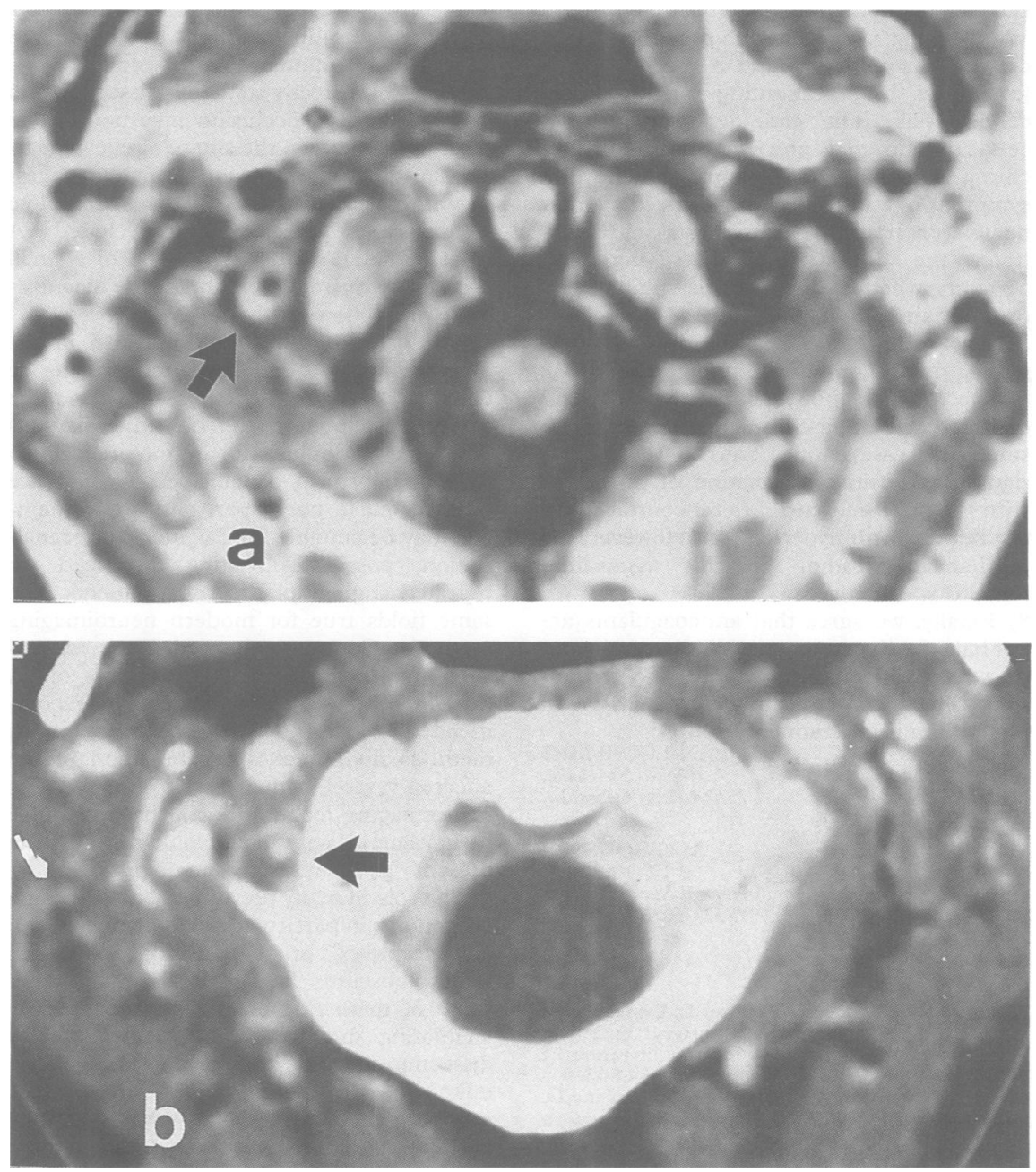

Figure Dissection of the third segment of the right vertebral artery.

a) Tl-weighted axial MRI (SE 500/25): Central hyposignal (flow void in the residual lumen of the vertebral artery) surrounded by hypersignal (parietal haematoma).

b) Thin section contrast-enhanced dynamic CT scan: Eccentric hyperdensity (injected residual lumen) surrounded by isodense haematoma and peripheral ring enhancement. 
can not only show "some abnormality that encourages angiographic examination" but can also diagnose dissections involving the pretransverse, C6-C5 and C5-C4 intertransverse segments of the VA. ${ }^{5}$ The diagnosis is based on the association of a localised increase in arterial diameter with haemodynamic signs of stenosis or occlusion and/or decreased pulsatility and intravascular echoes at the same level. Furthermore, ultrasonic examination is an excellent tool for the follow up of dissection.

6) Among other diagnostic procedures, the authors did not mention thin-section contrast-enhanced dynamic CT scan and MRI. By virtue of its sensitivity to both blood flow and thrombus formation, its multiplanar imaging capability, and its noninvasiveness, MRI (and soon MR angiography) is becoming the imaging modality of choice for the evaluation of suspected carotid or vertebral dissection (fig). At present, however, MRI does not assist in distinguishing between intraluminal and intramural thrombus and therefore does not allow the diagnosis of occlusive forms of vertebral dissection.

7) The relation of trauma to dissection is a complex issue. Hinse et $a^{1}$ considered their patient 4 as an example of traumatic (chiropractic manipulation) dissection. We recently reported $^{6}$ the case of a woman with a 3 week history of cervical pain who developed iscae$\mathrm{mia}$ in the basilar artery territory following cervical manipulation. Necropsy revealed 2 VA dissections, a recent one probably due to cervical manipulation and a second one, a few weeks old, accounting for the initial cervical pain. This case demonstrates that cervical pain that precedes and motivates chiropractic manipulation may be the first symptom of a hitherto unrecognised spontaneous (or traumatic) dissection and illustrates the difficulty in classifying with certainty whether dissection is spontaneous or traumatic.

Apart from trauma and fibromuscular dysplasia, other conditions implicated as risk factors for dissection include migraine, oral contraceptives, and chronic high blood pressure. In a case control study, ${ }^{7}$ we found a significant positive association of dissection with migraine and current oral contraceptive use but not with hypertension. However, the mechanisms leading to this association remains speculative.

8) Finally, we agree that anticoagulants are not harmful in extracranial VA dissection and may even be of benefit although no conclusion can be drawn from the comparison of nonrandomised treatment groups.

JEAN-LOUIS MAS Hopital Ste-Anne,
75674 Paris Cedex 14, France

M G BOUSSER

P J TOUBOUL

Hôpital Saint-Antoine

75571 Paris Cedex 12

France

1 Hinse P, Thie A, Lachenmayer L. Dissection of the extracranial vertebral artery: report of four cases and review of the literature. $\mathcal{F}$

2 Mas JL, Bousser MG, Hasboun D, Laplane D. Extracranial vertebral artery dissections: a review of 13 cases. Stroke 1987;18:1037-47.

3 Mokri B, Houser OW, Sandok BA, Piepgras DG. Spontaneous dissections of the vertebral arteries. Neurology 1988;38:880-5.

4 Hart RG. Vertebral artery dissection. Neurology 1988;38:987-9.

5 Touboul PJ, Mas JL, Bousser MG, Laplane D. Duplex scanning in extracranial vertebra artery dissection. Stroke 1987;18:116-21.
6 Mas JL, Henin D, Bousser MG, Chain F, Hauw JJ. Dissecting aneurysm of the vertebral artery and cervical manipulation: a case report with and cervical manipulation: a case repo
autopsy. Neurology 1989;39:512-5.

7 D'Anglejan-Chatillon J, Ribeiro V, Mas JL, Youl BD, Bousser MG. Migraine-A risk factor for dissections of cervical arteries. Headache 1989;29:560-1.

\section{Hinse and Thie reply:}

We thank Dr Mas and colleagues for their interest in our recent paper, ${ }^{1}$ and we appreciate the opportunity to comment on a few of the issues raised by them.

Our paper did not deal with the incidence of vertebral artery (VA) dissection which remains unknown. Better diagnosis and systematic study will hopefully shed more light on incidence of this condition in the future. In our analysis, we have included only case reports providing sufficient detail of the individual patient, but not summarised series, thus the results of Mokri et $a l^{2}$ were not considered. We apologise for not including the well documented patients by Mas et al. ${ }^{3}$

The question of internal carotid artery (ICA) dissection was not the subject of our paper. We agree that angiographic visualisation of all four brain-supplying vessels should be attempted in acute VA dissection in order not to miss concomitant asymptomatic ICA dissection. This point is of particular importance in spontaneous VA dissection: in 5 of 29 reviewed patients concomitant ICA dissection was documented, but not in 28 patients with traumatic VA dissection.

Mas et al correctly state that dissection as a cause of arterial occlusion may be hard to diagnose. However, the angiographic appearance of tapering occlusion is highly suggestive of dissection. ${ }^{4}$ In our patients, complete recanalisation of a formerly occluded VA (cases 2 and 3) and visualisation of a small pseudoaneurysm (case 2) made the diagnosis of VA dissection highly probable.

The value of ultrasound method in the diagnosis of VA dissection remains to be determined. Our own experience ${ }^{1}$ and the work of Touboul et $a l^{5}$ on three patients examined by duplex scan are too preliminary to allow any firm conclusions. As we have pointed out in our paper, ultrasound methods may be suitable for diagnosis of recanalisation, possibly obviating the need for repeated angiography in some patients. The same holds true for modern neuroimaging methods. Contrast-enhanced CT scan and MRI may corroborate the diagnosis of VA dissection, and are also increasingly recommended for follow up studies. However, both methods do not allow examination of the affected vessels in their entire length. These neuroimaging techniques will have to prove their value in a systematic study against the present "gold standard" (angiography).

The role of many presumably predisposing conditions, in particular migraine, use of oral contraceptives or hypertension, remains totally obscure. It is speculative whether some of these factors might act by merely facilitating the occurrence of stroke after dissection, but not dissection itself. It is also unknown why minor trauma may induce cervical dissections in some patients at any particular time, but why recurrences in these patients are rare.

P HINSE Department of Neurology University of Hamburg, Martinistr 52, 2000 Hamburg 20, Germany
1 Hinse P, Thie A, Lachenmayer L. Dissection of the extracranial vertebral artery: report of four cases and review of the literature. $f$ Neurol Neurosurg Pychiary 1991;54:863-9.

2 Mokri B, Houser OW, Sandok BA, Piepgras DG. Spontaneous dissections of the vertebral arteries. Neurology 1988;38:880-5.

3 Mas JL, Bousser MG, Hasboun D, Laplane D. Extracranial vertebral artery dissections: a review of 13 cases. Stroke 1987;18:1037-47.

4 Mokri B. Traumatic and spontaneous extrac ranial internal carotid artery dissections. $\mathcal{f}$ Neurol 1990;237:356-61.

5 Touboul PJ, Mas JL, Bousser MG, Laplane D. artery dissection. Stroke 1987;18:116-21.

\section{Late onset globoid cell leukodystrophy}

I read with great interest the paper by Grewal et al. ${ }^{1}$ I would like to add a few comments.

First, the authors suggest that their patient's late onset (at age 14) distinguishes his disease from globoid cell leukodystrophy (GLD) distinct from the infantile and late infantile onset types. This may be true for the first type, but the latter can occur within one family together with a later onset type. ${ }^{2}$

Second, it would be interesting to know whether the white matter hyperintensities on the MRI were diffuse or rather restricted to the occipito-parietal white matter, as described in other late onset GLD. ${ }^{2}$ If so, this posterior white matter involvement on MRI would seem to be very useful to distinguish GLD from other cerebral white matter diseases.

PETER VERDRU
Neurologie,
Universitaire Ziekenhuizen,
Herestraat 49,
3000 Leuven, Belgium

1 Grewal RP, Petronas N, Barton NW. Late onset globoid cell leukodystrophy. $\mathfrak{F}$ Neurol Neugloboid cell leukodystrophy. $\mathcal{F} N$
rosurg Psychiatry 1991;54:101 1-2.

2 Verdru P, Lammens M, Dom R, Van Elsen A, Carton H. Globoid cell leukodystrophy: a family with both late-infantile and adult type. Neurol 1991;41:1382-4.

3 Shapiro EG, Lockman LA, Krivit W. Correlation of Magnetic Resonance Imaging and Neuropsychological Abnormalities in Lateonset Leukodystrophies. Ann Neurol 1991; 30:466-7.

Aseptic meningitis associated with high dose intravenous immunoglobulin therapy

We read with great interest the report by Watson et al $^{1}$ and we would like to draw the authors attention to a similar case we published last year. ${ }^{2}$

Our patient was a seven year old boy with idiopathic thrombocytopenic purpura who had well-documented episodes of acute aseptic meningitis on two occasions after the second intravenous dose of immune globulin. On these two occasions, the patient developed aseptic meningitis on day three; quite identical to the two patients reported by Watson, whereas Kato's patient developed the aseptic meningitis two days after a five day course of intravenous immune globulin therapy. ${ }^{3}$

In our patient the immune globulin preparation used was Sandoglobulin IV (Sandoz), which is a formulation prepared by cold ethanol fractionation. It was given at a dose of $0.4 \mathrm{~g}$ per kilogram of body weight infused over a 11 hour period. 